\title{
26289 - ULTRASOUND TRANSVERSE APPROACH IS RELIABLE FOR LABOR EPIDURALS
}

\section{Cristian Arzola MD, Jose CA Carvalho, MD PhD; Cristian Arzola, MD; Sharon Davies, Ayman Rofaeel, MD Mount Sinai Hospital And University of Toronto, Toronto, ONTARIO, Canada}

INTRODUCTION: The efficacy and safety of epidural anesthesia relies on the accurate identification of the epidural space. Ultrasound imaging of the spine, using multiple scanning planes, has recently been proposed to facilitate this identification (1). The goal of this study was to assess the accuracy and precision of the transverse approach, used as a "single-screen" method, to facilitate labor epidurals.

METHODS: After REB approval and written informed consent, 61 patients requesting labor epidurals were enrolled. The ultrasound imaging was performed with the patient in the sitting position, at the L3/4 interspace, using the transverse approach, with a portable Titan Ultrasound System equipped with a 5.0-MHz curved array probe (Sonosite Canada Inc.). Using our proposed single-screen method, we identified the midline, the intervertebral space and the distance from the skin to the epidural space (ultrasound depth=UD). Epidural puncture was then performed in relation to these landmarks, and the distance from the skin to the epidural space was measured to the nearest half-cm of the gauged Tuohy needle (needle depth=ND). The accuracy of the insertion point was evaluated by the need to re-site and re-direct the needle during epidural puncture. The degree of agreement between UD and ND was calculated by the concordance correlation coefficient (CCC) (2). Bland-Altman analysis (3) was used to show the differences between UD and ND for each patient, with $95 \%$ limits of agreement. RESULTS: Maternal age was 33 $\pm 4.6 \mathrm{yrs}$, BMI 29.7 \pm 4.8 , UD $4.68 \pm 0.67 \mathrm{~cm}$ and ND $4.67 \pm 0.71 \mathrm{~cm}$. The CCC between UD and ND was 0.874 (95\% CI 0.814-0.934). The $95 \%$ limits of agreement were -0.683 to $0.702 \mathrm{~cm}$ (figure), and appeared to persist regardless of the BMI. The accuracy of the insertion point as defined by ultrasound was $87.7 \%$, with no need to re-direct the needle in $73.8 \%$ of the patients.

DISCUSSION: We found good accuracy in the ultrasound-determined insertion point and very good agreement between UD and ND, apparently unaffected by BMI. In $95 \%$ of the patients, the differences were $\pm 0.7 \mathrm{~cm}$. This suggests that our proposed ultrasound single-screen method using the transverse approach can be a reliable guide to facilitate labor epidural insertion, even in overweight patients.

REFERENCES: (1)Br J Anaesth 2001,86:798-804 (2)Biometrics 1989,45:255-268 (3)Lancet 1986 i:307-310 


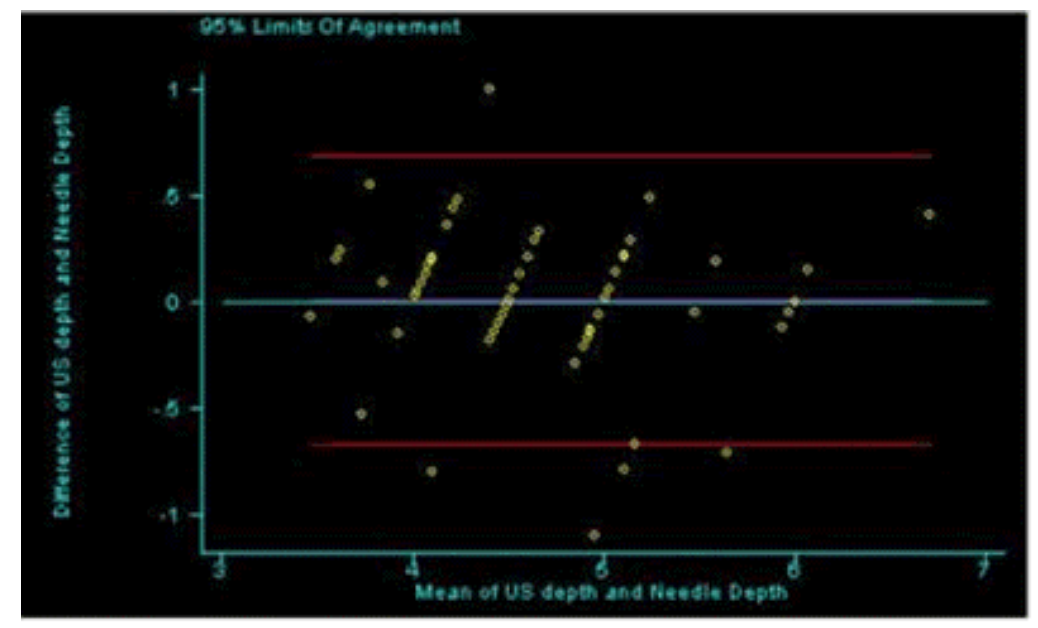

